\title{
De Canal Siete a ATC: dictadura, renovación tecnológica y apuesta por la televisión comercial (1978-1979)
}

Joaquín STICOTTI

\begin{abstract}
Resumo:
El presente artículo aborda cómo, durante la última dictadura cívico-militar argentina (1976-1983), el canal estatal ATC llega a liderar los índices de audiencia. Los preparativos del mundial de fútbol disputado en Argentina en 1978 constituyen la base de la modernización tecnológica de Canal 7 que pasa a llamarse ATC (Argentina Televisora a Color). Sin embargo, antes de las primeras transmisiones a color que comenzaron en 1980, a principios de 1979, ATC transforma su grilla de programación y su imagen gracias al empresario televisivo Carlos Montero. Y por primera vez en la historia, logra liderar los índices de audiencia. Este éxito se explica gracias a algunos programas de gran repercusión, elaborados en cooperación con civiles de larga trayectoria en el ámbito televisivo, pero también por una política cultural activa del gobierno militar que, además de invertir en tecnología, promueve la competencia entre los distintos canales bajo su control. Finalmente, la cooperación entre militares y civiles muestra sus limitaciones, en el contexto de ATC, precipitando el alejamiento de Montero.
\end{abstract}

Palavras-chave:

Televisión. Dictadura. Audiencia. Tecnología. Competencia.

\section{Do Canal 7 a ATC: ditadura, renovação tecnológica e aposta pela TV comercial (1978-1979)}

\section{Resumo:}

O presente artigo trata do modo em que, durante a última ditadura cívico-militar argentina (1976-1983), o canal estatal ATC (Argentina Televisora Color) chegou a liderar os índices de audiência. Os preparativos para a Copa do Mundo de 1978 disputada na Argentina formam a base da modernização tecnológica do Canal 7, que foi renomeado ATC. Contudo, antes das primeiras transmissões em cores que começaram em 1980, no início de 1979, a ATC transformou a sua grade de programação e a sua imagem graças ao empresário televisivo Carlos Montero. E pela primeira vez na história chegou a liderar os índices de audiência. O sucesso explica-se por programas de grande repercussão, elaborados em cooperação com cidadãos civis de vasta trajetória no âmbito televisivo, mas também em virtude de uma política cultural ativa do governo militar que, além de investir em tecnologia, fomentou a concorrência entre os diversos canais de televisão sob seu controle. Finalmente, a cooperação entre civis e militares revela as suas limitações, no contexto da ATC, o que precipita o afastamento de Montero.

Palavras chave:

Televisão. Ditadura. Audiência. Tecnologia. Concorrência.

\section{From Canal 7 to ATC: dictatorship, technology renovation and going for commercial television (1978-1979)}

\begin{abstract}
:
This article explores how, in the last argentinian dictatorship (1976-1983), the state channel ATC reaches the top ranking of audience. During the preparations for the football World Cup that takes place in Argentina in 1978, the state channel Canal 7 was modernized and changes the name to ATC (Argentina Televisora a Color). Although, before the first color transmissions in 1980, at the beginning of 1979, ATC changes its style and programmes by the work of the TV businessman Carlos Montero. And, for the first time, reaches the top ranks. This success is explained by some programmes with great reception elaborated by civilians with great careers in the TV show business, but also because of an active cultural

\footnotetext{
${ }^{1}$ Licenciado en Sociología por la Universidad de Buenos Aires, docente de Historia de los Medios y Sistemas de Comunicación en FSOC- UBA, cátedra Mirta Varela. Becario de CONICET y doctorando de la Facultad de Ciencias Sociales de la Universidad de Buenos Aires con el proyecto"Televisión nacional: programación, agentes estatales y hegemonía (1973-1989)”. joaquinsticotti@gmail.com
} 
politic led by the military government that includes technology investments and promotion of competence between the channels in their control. Finally, cooperation between militaries and civilians shows its limits in ATC producing the resignation of Carlos Montero.

Key words: Television, dictatorship, audience, technology, competence.

\section{INTRODUÇÃO}

La película Network (Sydney Lumet, 1976) es una sátira sobre el mundo de la televisión comercial que resulta ilustrativa respecto de la lucha por el bien más preciado dentro del mismo: el rating. En la película, tenemos dos modelos contrapuestos de empresarios televisivos: por un lado Max Schumacher (William Holden), antiguo editor de noticias, que representa el estereotipo del pionero participe de cierta bohemia de la televisión en los orígenes y dueño de principios éticos firmes a la hora de definir la programación. Por otro lado, Diana Chirstensen (Faye Donaway), ejemplo de la joven ejecutiva criada en el ambiente de la televisión, obsesionada con el rating como único modo de evaluar la pertinencia de un programa. Finalmente tenemos a Howard Beale (Peter Finch), histórico conductor del noticiero que, al borde de la locura psiquiátrica, comienza a realizar monólogos despotricando contra la sociedad contemporánea con un estilo iracundo. Los monólogos de Beale son un éxito de rating y hacen trepar a la emisora al tope de los rankings, un hecho festejado por Diana Christensen y objetado por Max Schumacher, amigo del conductor. Beale llega a desmayarse en cámara, pero nada detiene a Diana Christensen y el resto de los ejecutivos de UBS que mantienen el programa al aire. El límite llega solamente cuando los monólogos de Howard Beale comienzan a cuestionar el poder concentrado de algunas empresas estadounidenses y denunciar los negociados de la propia corporación mediática con capitales provenientes de Arabia Saudita. Sólo en ese momento, los ejecutivos de la plana mayor de la corporación cuestionan el programa de Beale y le hacen cambiar la fisonomía hasta hacerlo perder su carácter atractivo y su rating extraordinario.

Aunque Canal 7 - a diferencia del ficticio UBS - haya sido siempre un canal estatal, las tensiones en su interior se entretejieron, a lo largo de la historia, entre los mismos intereses: el propio poder estatal, la voluntad de los empresarios televisivos y la necesidad de adaptarse a las demandas del mercado de consumo.

Desde sus comienzos, Canal 7 se constituyó como un canal estatal con una programación comercial apoyada por la propaganda gubernamental (MINDEZ, 2001). No hubo, en la historia de Canal 7, un proyecto que pretendiera convertirlo en un canal cultural alternativo a lo comercial salvo las excepciones de las breves gestiones de 
Simonetti y Petrone en 1964 (VARELA, 2005) y la de Luis Pico Estrada en 1970 (MINDEZ, 2001).

Más allá de este perfil comercial, el primer y único momento en el que el canal logra liderar los índices de audiencia ocurrió en el marco de la última dictadura cívicomilitar, más precisamente durante la gestión de Carlos Montero entre el 3 de mayo y el 31 de diciembre de 1979. Montero le presenta al poder ejecutivo tres proyectos posibles: un canal cultural de bajo costo, un canal con una inversión limitada, como para estar tercero o cuarto en el rating, y un canal con una inversión significativa, como para pelear el primer puesto (ULLANOWSKY; SIRVEN; ITKIN, 2006). Los militares a cargo del ejecutivo eligen el tercero $y$, en pocos meses, el canal estatal que recientemente había cambiado su nombre a ATC (Argentina Televisora a Color) logra liderar por primera vez los índices de audiencia.

El presente artículo se propone explorar las condiciones de posibilidad y el desarrollo de esta breve, pero inédita, gestión del canal estatal argentino. Para esto será necesario, por un lado, remitirse a las transformaciones de infraestructura y tecnología ocurridas en Canal 7 a los fines de cubrir las transmisiones del mundial de fútbol realizado en Argentina en 1978. Por otro lado, a los cambios concretos en la grilla que, acompañados del nuevo soporte tecnológico, lograron instalar una programación comercial exitosa, a pesar de las tensiones entre los civiles responsables y los militares dirigentes. Teniendo sólo un antecedente que recorre la historia del Canal 7 desde 1953 a 2001 (MINDEZ, 2001) y ninguno que aborde específicamente el surgimiento de ATC, el presente artículo se propone como una primera aproximación exploratoria. Sin embargo, a modo de conclusión, podrán adelantarse algunos indicios en torno a la cooperación cívico-militar en el ámbito televisivo durante la última dictadura argentina (1976-1983) y algunas características del tipo de política cultural activa del gobierno dictatorial.

\section{Antecedentes: inversión y apuesta por la televisión comercial}

Partiendo de la caracterización de Basualdo (2011) se puede situar un cambio en el ciclo económico de la Argentina a partir del "Rodrigazo" ocurrido el 4 de junio de 1975. Se trató de una serie de aumentos dictados por el ministro Celestino Rodrigo que trajo como consecuencia una inflación de casi el $800 \%$ anual y una transferencia de ingresos del trabajo hacia el capital, devaluación mediante. El "Rodrigazo" abre un 
nuevo ciclo económico basado en la valorización financiera.

Este nuevo ciclo coincide con lo que Martín Becerra (2010) destaca como un cambio de etapa en la relación entre lo público y los medios de comunicación en nuestro país. La etapa que se abre en 1975 tiene, por un lado, la característica de una crisis en el modelo de autonomía relativa amenazada por el mercado y por otro lado, una retracción del consumo editorial, por la baja en el poder adquisitivo de los salarios, en beneficio del consumo de otros medios como la radio y la televisión.

En este marco, el 24 de junio de 1975, se sancionó la ley de expropiación que devolvía al estado las licencias de los canales públicos otorgadas en 1958 (MINDEZ, 2001). Esta caducidad de las licencias ya había sido determinada durante el gobierno dictatorial de Alejandro Agustín Lanusse. Las Fuerzas Armadas, nuevamente en el poder a partir del 24 de marzo de 1976, se dividieron la gestión de los canales equitativamente: Canal 9 quedaría para el Ejercito, Canal 11 para la Aeronáutica y Canal 13 para la Armada. Canal 7, el único que seguiría siendo estatal si se cumplía con la promesa de privatizar el resto en el mediano plazo, quedaba a cargo de la presidencia de la nación (MINDEZ, 2001).

Más allá de la defensa del liberalismo económico que los militares sostenían en muchos ámbitos, el control de los canales de televisión y las radios no era algo que estuviesen dispuestos a dejar fácilmente en manos del mercado. Esto puede explicarse por razones políticas: las violaciones a los derechos humanos cometidas por la dictadura requerían un blindaje mediático exhaustivo, difícil de confiar a un privado a cargo de un canal de televisión masivo (MASTRINI, 2005). Sin embargo, también había razones coherentes con la política económica de la dictadura para mantener este control sobre los medios. Como se dijo, se abría en Argentina, hacia 1976, un nuevo ciclo económico basado en la valorización financiera y una nueva etapa en la historia de los medios basada en la puesta en jaque de la autonomía relativa por parte del mercado. Esta nueva etapa, tanto nivel económico como social y cultural, requirió de una intervención activa del estado para imponer y garantizar la lógica de la libre competencia en todos los ámbitos. Las políticas culturales ligadas a la televisión estatal no fueron la excepción.

En los primeros meses de la dictadura, Canal 7 comenzó siendo la opción cultural en un sentido clásico (transmisiones de ballet, ópera, teatro) acompañada de las tradicionales transmisiones de fútbol y automovilismo. Pero pronto, ante la confirmación de otra herencia que había recibido la dictadura, se concibió al canal como 
una herramienta de una política cultural mucho más activa. Se trataba de la responsabilidad argentina de organizar, en 1978, el mundial de fútbol. La sede del mundial en Argentina fue ratificada a pesar de que, a nivel internacional, ya existían denuncias por las violaciones a los derechos humanos cometidas por la dictadura. Era, para los militares a cargo del ejecutivo, una ocasión ideal para mostrar la cara modernizadora del gobierno dictatorial y terminar con lo que ellos denunciaban como una "campaña anti argentina" orquestada desde el exterior (NOVARO; PALERMO, 2013).

En el caso del diseño de la señalética y la gráfica para el mundial, el gobierno tomó el trabajo que venía realizando un grupo de diseñadores influidos por la escuela alemana Ulm y por los juegos olímpicos de Múnich 1972 en los años previos (ALMEIDA, 2013). Por otro lado, la inversión en infraestructura fue de una gran magnitud. Además de la construcción de tres nuevos estadios en Córdoba, Mendoza y Mar del Plata, se construyó un nuevo megaestudio de televisión en la intersección de las calles Figueroa Alcorta y Tagle que luego pasaría a ser la nueva sede del Canal 7. El edificio fue realizado por el estudio de arquitectura MSGSSS, encabezado por Justo Solsona. El estudio tenía un prestigio reconocido a partir de la realización de otros edificios emblemáticos de la ciudad como la casa matriz del Banco Ciudad de Buenos Aires y el edificio Carlos Pellegrini. Los costos del mundial se calculan en 520 millones de dólares, de los cuales aproximadamente 100 se los llevó la construcción del nuevo megaestudio de Canal 7 (MINDEZ, 2001). Cuatro años después, España gastaría la cuarta parte de dicho presupuesto para organizar un mundial en su territorio.

El nuevo estudio del viejo Canal 7, que pasaría a llamarse ATC, tenía, además de un imponente edificio, tecnología de última generación para la transmisión televisiva. El equipamiento nuevo incluía máquinas de videotape - tecnología que comenzaba a utilizarse -, 30 cámaras nuevas, salas para post producción de audio y cuatro camiones de exteriores completos con usina eléctrica propia. A pesar de los esfuerzos por modernizar la infraestructura, la dictadura no logró su cometido de transmitir los partidos a color. El retraso en la renovación de los aparatos receptores determinó que los partidos se transmitieran en colores para el exterior mientras seguían siendo en blanco y negro para los televidentes argentinos.

Esta importante inversión en tecnología e infraestructura es central para comprender la decisión de la cúpula de las Fuerzas Armadas de apostar por el flamante 
ATC como el canal bajo su control que buscaría los índices más altos de audiencia. Sin embargo, se entiende que la tecnología no es el único elemento determinante de esta decisión. Se comprende, siguiendo a Williams (2011, p. 27), "la tecnología como algo buscado y desarrollado con determinados propósitos y prácticas en mente", en el marco de un proceso social total donde "no es marginal sino central" pero debe, para ser funcional a determinado interés, estar acompañada de un conjunto más amplio de decisiones políticas.

Así, la renovación de Canal 7, que respondía a la organización del mundial de fútbol pero también a una tendencia mundial del medio televisivo a transmitir en colores, se articuló también con la intención de orientar el uso de la nueva tecnología a construir una grilla de programación con perspectivas comerciales. $\mathrm{Y}$ en este caso, de un modo similar a lo sucedido con los diseñadores para la impronta gráfica del mundial (ALMEIDA, 2013) o a los arquitectos para la construcción del nuevo edificio del canal, los militares en el poder recurrieron, más que a hombres propios o afines políticamente, a quienes eran considerados los mejores y más exitosos en lo que hacían. Así, los ojos de los militares se posaron en Carlos Montero.

Montero venía de trabajar en Canal 13 junto al empresario cubano Goar Mestre. Cuando se determina la caducidad de las licencias de los canales, Montero permanece algunos meses más en el canal intervenido y más tarde renuncia por las dificultades para imponer un criterio propio (SIRVEN, 2013). Cuando le ofrecen la posibilidad de hacerse cargo del canal estatal, estaba por aceptar un cargo en la televisión brasileña al que renuncia para asumir el desafío en ATC.

\section{La gestión de Carlos Montero y los cambios en la programación}

A principios de 1979 Carlos Montero asume el cargo de gerente artístico del Canal 7, ya ubicado en las nuevas instalaciones de Figueroa Alcorta y Tagle. Es el mismo Montero quien propone la sigla ATC como nuevo nombre del canal y quién encarga la creación de un nuevo logotipo a la renombrada agencia de publicidad Lautrec, dirigida por Raúl Salles y Gianni Gasparini. El nuevo nombre y el logotipo tenían la finalidad de anunciar el esperado comienzo de la televisión a color, pero más allá de que las flamantes instalaciones del canal permitían la transmisión, aún no estaba resuelto el tema de la recepción. Los televisores a color comenzarán a extenderse lentamente recién a partir de 1980. 
A pesar de este retraso en la transmisión a color, las nuevas instalaciones de ATC permitían que las transmisiones tuviesen una mejor calidad de imagen que el resto de los canales. A partir de este favorable marco técnico, Montero se lanza a construir una nueva programación para el canal. Para esto debía establecer un diálogo constante, no exento de tensiones, con quién había sido nombrado interventor militar del canal, el Coronel Enrique Santos Paradelo. Es a Paradelo a quién Montero le presenta los tres proyectos de canal mencionados anteriormente y es el Coronel, orientado por una decisión política que probablemente viniera de jerarquías más altas, el que apuesta por el que apunta a liderar los índices de audiencia.

Montero conforma su equipo junto a dos experimentados gerentes de producción y programación que lo habían acompañado desde su época en Canal 13, ellos eran Enrique "Yuyo" Taboada y Juan Chotsourian, respectivamente. Desde el comienzo de su gestión logra negociar con el interventor una presencia mínima de programas proselitistas vinculados a los intereses de cada una de las Fuerzas Armadas que quedan limitados a la franja horaria que comenzaba a las 23 horas. Para el resto del armado de la grillla tuvo, según sus propias palabras, "poder de decisión total” (ULLANOWSKY; SIRVEN; ITKIN, 2006, p. 397) y un presupuesto abultado para poder seducir a una parte importante de las personalidades que conformaban el starsystem televisivo de la época. La nueva grilla de programación elaborada por Montero no solamente llevó a ATC a estar primero en los índices de audiencia por primera vez en su historia sino que dio sus frutos a nivel económico y transformó una facturación publicitaria que en marzo no llegaba a alcanzar los 70.000 dólares y en diciembre alcanzaría los 8.000 .000 (GENTE, 10 enero 1980). A pesar del gran éxito comercial, el consenso con los interventores militares se quebró al poco tiempo, precipitando el alejamiento de Montero y sus colaboradores en diciembre del mismo año en que había asumido.

\section{Cambios en la grilla: enlatados, clásicos y novedades}

Una de las características destacadas por los investigadores de la televisión de la época tiene que ver con el aumento del "enlatado" - programas grabados en el exterior - en detrimento de la producción nacional (VARELA, 2001). Este hecho se vincula al efecto del plan económico de Martínez de Hoz que condujo a un abaratamiento del dólar. En alguno casos, resultaba más rentable comprar un programa completo, producido en el extranjero, que encarar la producción de un programa a nivel local. Sin 
embargo es necesario, a la hora de analizar la rentabilidad de un programa, también prestar atención a su recepción y a su lugar en una grilla completa de programación. En el caso de la grilla de ATC elaborada por Montero se puede encontrar un equilibrio en la combinación de programas extranjeros, haciendo uso de la ventaja coyuntural del dólar barato, y la instalación de exitosos programas producidos en Argentina. El "enlatado" podía tener un espacio importante en la grilla, pero era necesario, para construir una programación de consumo masivo, apelar a algunos formatos locales que serían los puntales del éxito de la programación. Estos formatos serán el teleteatro y el noticiero.

En el caso del teleteatro, se trata de un género que podía tener éxito en su versión enlatada, pero que a la vez tenía una larga tradición local y costumbrista por detrás. Esta tradición se encuentra constituida por teleteatros que, aunque sea de un modo muy tangencial, están vinculados a la coyuntura político social del momento. Un ejemplo de esto lo podemos encontrar en el caso de Rolando Rivas, taxista (1972-1973) sobre el que se volverá más adelante. Este tipo de productos son casi imposibles de lograr con enlatados.

En el caso del noticiero, su carácter local resulta evidente. Sin embargo se presentaba como un desafío especialmente complejo por el particular atributo que se le reconoce a este tipo de programas: la verosimilitud. Los límites establecidos por la censura directa a la circulación de determinada información (VARELA, 2001) hacían muy difícil la creación, en un canal intervenido por la propia dictadura, de un noticiero verosímil: ¿cómo podría garantizarse la veracidad y sobre todo la no omisión de determinada información? Esto resultó uno de los principales desafíos para Montero.

Además del enlatado, que tuvo una presencia importante con las exitosas series El increíble Hulk y El chapulín colorado, la programación nueva podría sintetizarse a partir de tres categorías que se explorarán con mayor profundidad: un programa que traía su propia audiencia masiva de otro canal: Almorzando con Mirtha Legrand; el noticiero, que se llamó 60 minutos y, finalmente, los teleteatros más exitosos, que fueron Andrea Celeste y Los hijos de López.

\section{Un clásico con éxito garantizado: Almorzando con Mirtha Legrand}

Almorzando con Mirtha Legrand, que se emitía desde 1969 en distintos canales, era un éxito ya probado en 1979. Producto de esto, resultó uno de los primeros objetivos de Montero para construir la base de una programación exitosa desde el punto de vista 
comercial. A partir de este ejemplo, se puede comprender la importancia de estudiar la grilla de programación como una totalidad de elementos que se encuentran relacionados. Para asumir el riesgo de algunos programas cuyo éxito no estaba garantizado - como los teleteatros y el noticiero - Montero debe recurrir a éxitos probados que le garanticen un piso de audiencia como pueden ser algunos programas enlatados (el éxito de Chespirito ya había sido probado con la enorme audiencia de la que gozaba El Chavo del ocho) y clásicos de otros canales como el programa conducido por Mirtha Legrand. En una entrevista otorgada a la revista Gente el 10 de enero de 1980, Montero se atribuye el mérito de haber logrado traer este programa a ATC: "Mirtha Legrand fue mi primera obsesión. Ya tenía un contrato firmado con Canal 13 y lo cambió, por la misma plata, sólo porque creyó en mí".

El programa consistía y consiste (ya que se sigue emitiendo, siendo el más longevo de la televisión argentina) en un almuerzo con diferentes figuras del espectáculo y la farándula local. La conductora - Mirtha Legrand - generalmente hace preguntas a los invitados a cerca de sus trayectorias y sus actividades actuales. En una extensa crónica publicada en el diario Clarín el 4 de mayo de 1979, se cuentan detalles de la grabación, en vivo, del primer programa del ciclo que se emitía por primera vez en ATC. La crónica afirma que la conductora llega a las 9:30 de la mañana y no sale de su camarín hasta quince minutos antes de las 14 horas. También relata que todas las instalaciones del flamante edificio de ATC se encuentran dedicadas a este programa y que la primera entrega del ciclo se vive con gran nerviosismo por parte de todo el equipo técnico, los asistentes y los cocineros a cargo del almuerzo. Antes de comenzar su programa, Mirtha Legrand sale de su camarín a la calle Figueroa Alcorta y realiza una entrada formal, ya lista para empezar el programa. Recorre las instalaciones del canal y recibe cálidos saludos por parte de los numerosos empleados hasta llegar al estudio y saludar a sus invitados en esa ocasión especial. La nota aclara que, más allá del equipo estable que realiza el programa, "todo el personal de la televisora que sea necesario podrá ser utilizado a los fines de la realización” (CLARÍN, 4 mayo 1979, p. 1, Suplemento Espectáculos). Está claro que Almorzando con Mirtha Legrand es la primera gran apuesta de Carlos Montero para darle a ATC un primer paso hacia el éxito en la televisión comercial.

\section{Un nuevo noticiero: 60 minutos}


La idea del noticiero fue concebida por Montero, quien ya había creado un formato similar y muy exitoso con Telenoche en el Canal 13. Resulta clara la conexión con el noticiero norteamericano 60 minutes de la cadena CBS. Gracias a su trabajo en Canal 13, junto al empresario cubano Goar Mestre, Montero había pasado unos meses en las ciudades de Nueva York y San Francisco recorriendo estudios de televisión y grabaciones de programas (SIRVEN, 2013). Es posible que muchas de las ideas de formato de 60 Minutos hayan resultado inspiradas por este viaje. Sin embargo, el noticiero tenía, también, una impronta propia, local, que mezclaba figuras que ya tenían una trayectoria en el medio televisivo con algunos jóvenes que recién comenzaban.

El noticiero comienza yendo en el segmento 20 a 21 horas, pero hacia mitad de año pasa al de 21 a 22, probablemente para competir con el noticiero de Canal 13 llamado Mónica Presenta, paradójicamente otra creación del propio Montero, conducido por Mónica Cahen D’Anvers. De junio a agosto, el rating de 60 minutos aumenta (junio:7 puntos; julio: 9,2 puntos y agosto: 21,8 puntos) mientras que el de Mónica presenta baja (junio: 17,5; julio: 12,3; agosto: 12,6). Se pueden proponer tres razones de este desplazamiento: en primer lugar, el noticiero de ATC contaba con la mencionada infraestructura heredada de las reformas ocurridas en las instalaciones del canal con motivo del mundial de 1978. Esta herencia, le había dejado, por ejemplo, seis cámaras portátiles TK 76-RCA muy útiles para la dinámica de un noticiero con móviles en distintos lugares de la ciudad, el interior e incluso fuera del país. La infraestructura del Canal 13 no era para nada despreciable, y el noticiero también tenía tecnología para grabar en exteriores. Pero hay que agregar que 1979 coincide con una serie de reformas urbanas planteadas por el gobierno militar que obligaron a Canal 13 a remodelar su edificio ubicado en el barrio de Constitución, producto de una autopista urbana que se construyó muy cerca de sus instalaciones. Estas reformas complicaron el funcionamiento del canal (NIELSEN, 2006).

En segundo lugar, se debe considerar el propio saber hacer de Carlos Montero en el ámbito de la televisión comercial para lograr convertir, en una época de marcada censura a la información, a un noticiero en un éxito comercial. Así lo veía él mismo en una entrevista otorgada a la revista Somos, el 7 de septiembre de 1979: "60 minutos se impone por presentar caras nuevas para la TV (Betty Elizalde, Fernando Bravo, María Larreta, Silvia Fernández Barrios y José Gómez Fuentes). Tiene ritmo de diario, ya que su único tema es la actualidad: la vedette es la noticia, no los cronistas". 
Repasando algunas historias de la televisión en Argentina (NIELSEN, 2006; ULLANOWKSY; SIRVEN; ITKIN, 2006)), el recuerdo del noticiero se vincula más a la época de la Guerra de Malvinas (1982), cuando el régimen militar se encontraba en una profunda crisis de legitimidad y el noticiero, en la voz de de José Gómez Fuentes, insistía con la poco creíble idea de "Vamos ganando". Las cosas eran muy distintas para 1979. Tomando las palabras de otra de sus protagonistas, en una entrevista reciente: "Al principio, en ATC, a Paradelo lo odiábamos porque se había bajado de un tanque. Y terminó siendo muy buen tipo, entró a saber de televisión. Nunca hubo un ATC como el de esa época y nunca lo volvió a haber" (BARRIO, 27 feb. 2013).

En tercer lugar, se puede el éxito de 60 minutos a una productiva cooperación de militares con civiles provenientes del campo de la televisión comercial. La estrategia ganadora de Montero no hubiese sido posible sin tener al coronel interventor de su lado. Aunque parte de la estrategia consistía también en sostener, a pesar de que todos los canales estuviesen controlados por las Fuerzas Armadas, la competencia entre los mismos. El éxito de 60 minutos no se basó en que era el único noticiero que se podía ver sino en que era el más visto en el marco de la competencia restringida que las autoridades militares proponían con sus diferentes canales de televisión intervenidos.

\section{Dos teleteatros exitosos: Los hijos de López y Andrea Celeste}

Una particularidad de la programación de ATC en 1979 fue la de tener, simultáneamente en el aire, dos teleteatros muy exitosos. Este fenómeno explica en buena parte el hecho de que haya podido llegar a liderar los índices de audiencia por única vez en su historia. Los programas eran: Los hijos de López (que comenzó yendo en el segmento de 21 a 22 horas y pasó, con el cambio de 60 minutos, al segmento de 20 a 21 horas) y Andrea Celeste (de 19 a 20 horas).

Los hijos de López era un teleteatro escrito por Hugo Moser, autor de uno de los primeros - y más exitosos - teleteatros argentinos llamado La familia Falcón (emitida entre 1962 y 1969 por el Canal 13). Moser era, al igual que Montero, un hombre que se había consolidado en el ambiente de la televisión comercial en el Canal 13 de Goar Mestre. Cuando desembarca en ATC ya tenía una fama y un lugar consolidado. Sin embargo, Los hijos de López le supuso un nuevo desafío en lo formal: el guión del teleteatro se escribía día por día, incorporando a la historia los hechos cotidianos. Esto resultaba posible, en gran medida, por la infraestructura del canal y el despliegue 
técnico dedicado al programa que incluía 7 camarógrafos, 4 sonidistas, 3 operadores de video-tape e incluso un camión de exteriores, de uso poco frecuente en teleteatros anteriores. Además, esta modalidad le suma una impronta local y coyuntural al argumento del programa, llevando más lejos un procedimiento ya inaugurado en el mencionado teleteatro Rolando Rivas, taxista, escrita por Alberto Migré y emitida por Canal 13 entre 1972 y 1973. En esta telenovela, además de la búsqueda de dar con cierta sensibilidad propia de la clase media, se incorporan elementos coyunturales como la inminente asunción de Cámpora el 25 de mayo de 1973 (CARASSAI, 2013). La posibilidad de escribir los guiones día a día permite que Los hijos de López lleve más lejos éste procedimiento, incorporando los hechos coyunturales casi en "tiempo real". Un ejemplo de esto se puede ver con la pelea por el título mundial de peso mediopesado que enfrentó a Víctor Galíndez con Marvin Johnson en Nueva Orleans el 30 de noviembre de 1979: la telenovela incorpora el acontecimiento de la pelea en la misma semana en que esta iba a realizarse.

Más allá de la modalidad técnica de escribir los guiones día a día, los textos de Moser no podían incorporar cualquier tipo de acontecimiento. Las referencias a la política, muy presentes en Rolando Rivas, taxista eran difíciles de imaginar en 1979. Sin embargo, las dificultades de la censura no se limitaban a las cuestiones políticas sino que también se extendían, como se sabe por otras expresiones artísticas, a la moral y a las llamadas "buenas costumbres". En una entrevista a la revista Somos (7 dic. 1979), Moser afirma sobre el teleteatro: "Es solamente una telecomedia y punto. No pretende reflejar a la familia argentina". Justificando el fin del ciclo y su alejamiento de ATC para diciembre, agrega: "Hablando a calzón quitado, la plata ya no me compensa... hay problemas y uno se cansa" (SOMOS, 7 dic. 1979).

Según se puede deducir, este malestar viene producto de ciertas críticas realizadas por parte de los militares al contenido del teleteatro que incluía un personaje, interpretado por Leonor Benedetto, que hacía explícitas algunas fantasías sexuales. En su libro sobre la historia de la televisión argentina, Ullanowsky, Sirven e Itkin (2006) agregan que, durante las grabaciones de Los hijos de López, los militares mandan gente a supervisar los rodajes y comienzan a proponer proyectos alternativos para reemplazar el teleteatro. Es probable que este tipo de restricciones hayan sido las que determinaron el alejamiento de Los hijos de López de la pantalla de ATC a fines de 1979 así como el alejamiento del propio Carlo Montero de la dirección de la emisora. El consenso cívico 
militar exponía en ATC su carácter frágil.

Con el teleteatro Andrea Celeste, escrito por Abel Santa Cruz, sucedió algo similar. Su éxito comercial resultaba innegable (hacía incluso más puntos de rating que Los Hijos de López, llegando a marcar picos de 30 puntos que representaban más de dos millones de espectadores diarios) y era una parte central de la programación ideada por Montero. Además, se repetía en varias provincias del interior de la Argentina, por medio de los canales locales. El argumento consistía en una niña huérfana (Andrea del Boca) que era adoptada por un hombre viudo (Alberto Argibay). Esto despierta prohibiciones dictadas por los interventores de las provincias de Catamarca, Mendoza y La Rioja con la justificación de que del teleteatro "surgían algunos rasgos negativos para la mentalidad infantil" (SOMOS, 28 sept. 1979). Buscando tomar distancia de este conflicto, Montero replicará a la misma revista lo siguiente: "Vendemos entre 2000 y 2500 horas de programación mensuales a más de 20 canales del interior. Ellos son dueños de utilizarlas como mejor les parezca porque el compromiso es de horas y no de programas (...)” (SOMOS, 28 sept. 1979). En este comentario se puede ver, al mismo tiempo, el pragmatismo del director de ATC para sortear determinados conflictos pero también el germen de objeciones morales que, probablemente, precipitaron su salida a fines de 1979.

\section{Conclusiones}

A partir de lo expuesto, se pueden identificar dos momentos en las relaciones cívico militares al interior de ATC durante 1979: un primer momento de consenso, donde el nuevo equipo liderado por Montero puede actuar sin restricciones (presupuestarias o de contenido) avanzando en su proyecto de un canal comercial que aspira - y logra - liderar los índices de audiencia. Y un segundo momento donde dicho consenso se quiebra producto de determinadas opiniones militares que circulan criticando, fundamentalmente, a los teleteatros Andrea Celeste y Los hijos de López.

Sin embargo, indagando en las razones del quiebre del consenso, no se encuentran razones políticas sino diferencias en cuanto a criterios morales de lo que debía o no mostrarse en televisión. Una razón más para sostener la ausencia de diferencias políticas puede resultar el hecho de que el programa cuyo contenido era más susceptible de ser explícitamente politizado, como es el noticiero no solamente estuvo exento de críticas y objeciones sino que se mantuvo en los años posteriores luego del 
alejamiento de Montero de la emisora.

Por otro lado, pensando en la política cultural llevada adelante por los militares, se puede notar cierta búsqueda, en el marco de la televisión, basada en la meritocracia del éxito comercial más que en el mérito acumulado en el campo cultural. En esto, se puede esbozar una diferencia con Brasil, donde, siguiendo la hipótesis de Sacramento (2012), las transformaciones en la TV Globo apelaron al "buen gusto" de cierta izquierda cultural para armar la nueva programación.

Finalmente, lo que se puede sacar en limpio del análisis propuesto es que para desarrollar su política televisiva el gobierno dictatorial se sostuvo en dos pilares fundamentales: la inversión en tecnología e infraestructura y la apelación a civiles con una probada trayectoria exitosa en la televisión comercial. La modalidad para llevar adelante su objetivo fue la de reforzar el presupuesto de un canal armando una grilla de programación que combinará éxitos probados con innovaciones prometedoras. También, sostuvo el presupuesto del resto de los canales, manejados por las distintas fuerzas, creando un clima de competencia donde ATC se irguió por primera vez como el canal con mayores índices de audiencia.

El objetivo siempre fue, como marca la contemporánea película Network respecto del campo televisivo, un nivel alto de rating. Las diferencias entre civiles y militares se manifestaron cuando determinadas objeciones morales no permitieron la continuidad de los proyectos de ficción más exitosos.

Submetido em 13.11.2016

Aceito em 14.04.2017

\section{REFERÊNCIA BIBLIOGRÁFICA}

ALMEIDA, Marta. Argentina '78 World Cup and the echoes of México '68: Internacionalism and Latin American Design. An intellectual history of design in the 1960s and 1970s: Politics and Periphery. Journal of Design History, v. 27, n. 1, p. 5875, Dec. 2013. Published by: Oxford University Press.

BARRIO, Silvia Fernández. La Nación, 27 fev. 2013. Consultado en: $<$ goo.gl/BrGJnN>.

BASUALDO, Eduardo. Sistema político y modelo de acumulación. Tres ensayos sobre la argentina actual. Buenos Aires: Atuel, 2011.

BECERRA, Martín. Las noticias van al mercado: etapas de intermediación de lo público en la historia de los medios de la Argentina. En: LUGONES, Gustavo; 
FLORES, Jorge. (Comp.) Intérpretes e interpretaciones de la Argentina en el bicentenario. Buenos Aires: Universidad Nacional de Quilmes, 2010, p. 139-165.

CARASSAI, Sebastián. Los años setenta de la gente común. La naturalización de la violencia. Buenos Aires: Siglo Veintiuno Editores, 2013.

MASTRINI, Guillermo (editor). Mucho ruido, pocas leyes. Economía y política de comunicación en la Argentina (1920-2004). Buenos Aires: La Crujía, 2005.

MINDEZ, Leonardo. Canal 7: medio siglo perdido: la historia del Estado argentino y su estación de televisión. Buenos Aires: La Crujía, 2001.

NIELSEN, Jorge. La magia de la televisión argentina. Cierta historia documentada (Tomo III). Buenos Aires: Ediciones del Jilguero, 2006.

NOVARO, Marcos; PALERMO, Vicente. La dictadura militar 1976/1983. Del golpe de Estado a la restauración democrática. Buenos Aires: Paidós, 2013.

SACRAMENTO, Igor. La televisión brasilera en los años de la dictadura militar (19641984): la higienización del grotesco como afirmación de lo moderno. En: Rehime. Cuadernos de la Red de Historia de los Medios, Buenos Aires, año 2, n. 2, p. 52-101, 2012. Editor responsable: Cátedra de Historia de los Medios, Facultad de Ciencias Sociales, UBA. Disponible en: $<$ http://www.rehime.com.ar/escritos/cuadernos/ReHiMe_Cuaderno_02_final_web_02.p df $>$.

SIRVÉN, Pablo. El rey de la TV. Goar Mestre y la pelea entre gobiernos y medios latinoamericanos. De Fidel Castro a Perón. Buenos Aires: Sudamericana, 2013.

SOMOS. Entrevista a Carlos Montero. Buenos Aires, 28 sept. 1979.

SOMOS. Entrevista a Hugo Moser. Buenos Aires, 7 dic. 1979.

SOMOS. Entrevista a Carlos Montero. Buenos Aires, 7 de sept. 1979.

ULANOVSKY, Carlos; ITKIN, Silvia; SIRVÉN, Pablo. Estamos en el aire. Buenos Aires: Emecé, 2006.

VARELA, Mirta. La televisión criolla. Desde sus inicios hasta la llegada del hombre a la luna 1951-1969. Buenos Aires: Edhasa, 2005.

VARELA, Mirta. Silencio, mordaza y optimismo. Revista Todo es Historia, Buenios Aires, año XXXIV, n. 404, p. 50-63, marzo 2001.

WILLIAMS, Raymond. Televisión. Tecnología y forma cultural. Buenos Aires: Paidós, 2011. 